$g, v_{\mathrm{D}}, \mu$ and $v$ are the number of excited electrons $\mathrm{cm}^{-3} \mathrm{sec}^{-1}$, the drift velocity, the mobility and the thermal velocity, respectively. In Fig. 2 the lines are theoretical curves according to equations (4), (1) and (3). As to the four upper curves there is good agreement of the measured data with a field- and temperature dependence according to $(1 \mathrm{a})$. The parameter $p_{\mathrm{r}} / \mu$ is adjusted to fit the data. While recording such a set of measurements the density $p_{\mathrm{r}}$ of positive centers is increased by the irradiation ${ }^{3,7}$, and thus $p_{\mathrm{r}}$ is somewhat different from curve to curve even for the same sample. Taking that into account, we get temperature independent values for $p_{\mathrm{r}} / \mu$. The density $p_{\mathrm{r}}$ is assumed to be constant, since most of the states are as deep as or deeper than $2 \mathrm{eV}$ below the conduction band edge ${ }^{6}$. We arrive at the conclusion that the mobility $\mu$ is not or nearly not temperature dependent. Our measurements agree with Goodman's value of $\mu=29 \mathrm{~cm}^{2}$ $/ \mathrm{Vsec}^{8}$, measured at $300^{\circ} \mathrm{K}$. Therefore we conclude that the mobility in fused silica is determined by elastic scattering at about $3 \cdot 10^{19} \mathrm{~cm}^{-3}$ neutral impurities. Mean values of $p_{\mathrm{r}}$ are given in Fig. 2 .

In Fig. 2 the lower curve at $8{ }^{\circ} \mathrm{K}$ is calculated with cross section $(1 \mathrm{~b})$, with a value of $p_{\mathrm{r}} / \mu$ derived from the $77^{\circ} \mathrm{K}$-curve of this sample, and with $\lambda_{\mathrm{p}}=106 \AA$.

7 J. P. Mitchell, IEEE Trans. Electron Devices ED-14, No. 11, 764 [1967].

8 A. M. Goodman, Phys. Rev. 164, 1145 [1967].
The rate of energy loss derived from this mean free path for loss of a phonon is much greater than that for crystalline $\mathrm{SiO}_{2}$, estimated from the formulas for piezoelectric or acoustic deformation potential scattering ${ }^{9}$. It is assumed that in disordered solids a different energy loss mechanism is present.

At high voltages two saturation effects appear, limiting the further increase of the induced current. Besides the schubweg saturation, found in photoconductors with blocking contacts when the schubweg $\tau v_{\mathrm{D}}$ (4) becomes comporable with the sample thickness, the drift velocity $v_{\mathrm{D}}$ becomes constant on the level of the velocity of sound $v_{\mathrm{s}}$. This effect known as acoustoelectric effect from piezoelectric semiconductors ${ }^{9,10}$ starts at about $v_{\mathrm{S}} / \mu=1.9 \cdot 10^{4} \mathrm{~V} / \mathrm{cm}$. In some samples especially at lower temperatures the effect is accompanied with fluctuations of the induced current and with charge redistributions within the sample. Further results and details of the measurements will be published elsewhere ${ }^{3}$.

The author wishes to thank Prof. Dr. E. BAGGE for his encouragement and generous support, and Dipl.-Phys. K. BL̈̈NSDORF and Dr. U.-J. Roose for continuous help and useful discussions.

9 A. Rose, Helv. Phys. Acta 41, 1119 [1968] ; RCA-Rev. 27,
98, 600 [1966] ; RCA-Rev. 28,634 [1967]. 10 A. R. Moore, J. Appl. Phys. 38, 2327 [1967].

\section{Die Zustandsgleichung isotoper Äthylene}

\section{Th. Dorfmüller und W. Göpel}

Institut für Physikalische Chemie und Elektrochemie der Technischen Universität Hannover

(Z. Naturforsch. 24 a, 852-854 [1969] ; eingegangen am 14. März 1969)

The second virial coefficients of $\mathrm{C}_{2} \mathrm{H}_{4}$ and $\mathrm{C}_{2} \mathrm{D}_{4}$ have been measured at $236.04{ }^{\circ} \mathrm{K}$. The experimental isotope effect of $1.07 \%$ can be separated into one contribution from a translational quantum effect and one contribution which is due to the different molar volumes of the isotopes. In this temperature region the isotope effect is shown to be mainly a volume effect. This explanation is confirmed by results from molar volume measurements of the liquid isotopes.

Im Rahmen einer Untersuchung über die Zustands. gleichung isotopensubstituierter Substanzen wurden die 2. Virialkoeffizienten der deuterierten Äthylenisotope gemessen. An dieser Stelle wird über die ersten Ergebnisse für die Isotope $\mathrm{C}_{2} \mathrm{H}_{4}$ und $\mathrm{C}_{2} \mathrm{D}_{2}$ berichtet.

Vergleichende Messungen der 2. Virialkoeffizienten isotoper Gase liegen lediglich für die Paare $\mathrm{H}_{2}-\mathrm{D}_{2}{ }^{1}$ und $\mathrm{CH}_{4}-\mathrm{CD}_{4}{ }^{2}$ vor.

1 F. V. Varekamp u. J. M. Beenakker, Physica 25, 889 [1959].

\section{Experimentelles}

Für die Messungen wurde im Prinzip das Verfahren von BurnetT ${ }^{3}$ verwendet. Hierbei wird ein Gas in einen von zwei über ein Ventil verbundenen Metallbehälter eingelassen und der Druck $p_{1}$ gemessen. Nach Expansion in das zweite Volumen stellt sich ein Druck $p_{2}$ ein, der zum Druck $p_{1}$ in einem Verhältnis steht, das bei idealen Gas-Bedingungen dem umgekehrten Verhältnis der von dem Gas eingenommenen Volumina gleich sein wird. Verhält sich das Gas nicht ideal, so weicht $p_{1} / p_{2}$ von dem Verhältnis der Volumina ab. Die Abweichung des Druckquotienten bei einer Reihe von sukzessiven Expansionen ermöglicht eine sehr genaue Absolutbestimmung der Virialkoeffizienten des Gases. Wegen der Kleinheit der Effekte waren die Anforderungen an die Genauigkeit der Druckmessungen und an die Temperaturkonstanz während einer Expansionsserie sehr groß. So wurde der Druck bei 300 Torr auf sechs Stellen genau gemessen und die Temperatur auf $2 \cdot 10^{-3}{ }^{\circ} \mathrm{K}$ genau über mehrere Stunden geregelt. Weitere Angaben über die technische Ausführung der Apparatur werden an anderer Stelle veröffentlicht.

2 G. Thomaes u. R. Van Steenwinkel, Trans. Faraday Soc. 57, 307 [1961].

3 E. Burnetr, J. Appl. Mech. 58, 136 [1936]. 


\section{Meßergebnisse}

Die Messungen wurden zunächst an den beiden Äthylenisotopen $\mathrm{C}_{2} \mathrm{H}_{4}$ und $\mathrm{C}_{2} \mathrm{D}_{4}$ durchgeführt.

Bei einer Meßtemperatur von $236,04{ }^{\circ} \mathrm{K}$ wurden folgende Werte für die Virialkoeffizienten erhalten. Die gestrichenen Größen beziehen sich in diesem Text immer auf das leichte und die ungestrichenen auf das deuterierte Isotop.

$$
\begin{aligned}
B^{\prime} & =-242,2 \pm 0,3 \mathrm{~cm}^{3} / \mathrm{Mol}, \\
B & =-239,6 \pm 0,3 \mathrm{~cm}^{3} / \mathrm{Mol} .
\end{aligned}
$$

Der Isotopieeffekt beträgt somit:

$$
\frac{B^{\prime}-B}{B^{\prime}}=+(1,07 \pm 0,12) \cdot 10^{-2} .
$$

Der Betrag des zweiten Virialkoeffizienten ist demnach bei dem schweren Isotop um etwa $1 \%$ kleiner als bei dem leichten.

\section{Diskussion der Ergebnisse}

Für das Auftreten von Isotopieeffekten bei der $\mathrm{Zu}$ standsgleichung von Gasen können hauptsächlich folgende Effekte verantwortlich gemacht werden.

a) Intermolekulare Translations-Quanteneffekte,

b) Intermolekulare Rotations-Quanteneffekte,

c) Intramolekulare Quanteneffekte, die sich als Unterschiede der Parameter des intermolekularen Potentials äußern.

Die intermolekularen Quanteneffekte können für nicht zu tiefe Temperaturen mit Hilfe einer Reihenentwicklung nach Potenzen von $h^{2}$ entwickelt werden ${ }^{4}$. Diese Entwicklung hat für den zweiten Virialkoeffizienten die Form:

$$
\begin{aligned}
B(T)=B_{\mathrm{kl}}(T) & +\left(h^{2} / m\right) B_{1} \operatorname{tr}(T)+\left(h^{2} / m\right)^{2} B_{2} \operatorname{tr}(T)+\ldots, \\
& +\left(h^{2} / I\right) B_{1}{ }^{\text {rot }}(T)+\left(h^{2} / I\right)^{2} B_{2}{ }^{\text {rot }}(T)+\ldots
\end{aligned}
$$

In der Beziehung (1) stellen $m$ und $I$ die Masse bzw. das Trägheitsmoment des betreffenden Moleküls dar.

Der klassische Term $B_{\mathrm{kl}}(T)$ wird durch die bekannte Beziehung

$$
B_{\mathrm{kl}}(T)=-2 \pi N \int_{0}^{\infty}\left(e^{-\varphi(r) / k T}-1\right) r^{2} \mathrm{~d} r
$$

gegeben, die den Zusammenhang zwischen Paarpotential $\varphi(r)$ und dem zweiten Virialkoeffizienten zeigt.

In die Translations-Quantenkorrekturen gehen außer dem Wert des Potentials dessen Ableitungen nach dem intermolekularen Abstand $r$ ein. Die Translationsterme $B_{1}{ }^{\operatorname{tr}}(T)$ und $B_{2}{ }^{\operatorname{tr}}(T)$ sind für das $12-6$-Lennard-JonesPotential

$$
\varphi(r)=-4 \varepsilon\left[(\sigma / r)^{12}-(\sigma / r)^{6}\right]
$$

tabelliert worden ${ }^{5}$. Somit war eine Abschätzung des Isotopieeffekts für die Translationsquantenkorrektur

4 G. Uhlenbeck u. E. Beth, Physica 5, 945 [1936].

5 J. Hirschfelder, C. Curtiss u. B. Bird, Molecular Theory of Gases and Liquids, Wiley, New York 1953. möglich. Im vorliegenden Fall ergibt sich ein Isotopieeffekt von $0,12 \mathrm{~cm}^{3} / \mathrm{Mol}$

Die Rotationsterme $B_{1}{ }^{\text {rot }}(T), B_{2}{ }^{\text {rot }}(T), \ldots$ treten nur bei solchen Molekülen auf, bei denen das intermolekulare Potentiatl orientierungsabhängig ist, was beim Äthylenmolekül auch zutrifft. Zur Berechnung dieser Terme, in die die Ableitungen der Potentialfunktion nach den Orientierungswinkeln eingehen, muß ein winkelabhängiges Potential $\varphi\left(r, \vartheta_{1}, \vartheta_{2}, \varphi\right)$ für das betreffende Molekül bekannt sein. Im Falle des Äthylens ist die Aufstellung einer solchen Potentialfunktion nicht ohne weiteres möglich, jedoch konnte mit einer einfachen Winkelabhängigkeit nach $\cos ^{2} \vartheta$ abgeschätzt werden, daß der Isotopieeffekt auf Grund dieses Effekts vernachlässigbar klein ist, wenn die Isotopensubstitution keine Symmetrieänderung des Moleküls zur Folge hat. Dies ist der Fall bei $\mathrm{C}_{2} \mathrm{H}_{4}-\mathrm{C}_{2} \mathrm{D}_{4}$.

Der intramolekulare Quanteneffekt bewirkt eine Wechselwirkung der gequantelten inneren Freiheitsgrade des Moleküls auf die Translations-Freiheitsgrade, die in erster Näherung mit Hilfe einer Paarpotential-Wechselwirkung Gl. (3) klassisch beschrieben werden können [Gl. (2)]. In dieser Näherung kann man denjenigen Anteil des Isotopieeffekts, der nicht auf intermolekulare Quanteneffekte zurückzuführen ist, durch eine Modifizierung der Parameter $\varepsilon$ und $\sigma$ des Paarpotentials beschreiben.

Eine Isotopensubstituierung hat eine Verschiebung der Schwingungsniveaus und somit eine Änderung der mittleren Entfernung der schwingenden Atome zur Folge. Ersetzt man beispielsweise im Äthylen die HAtome durch D-Atome, so beobachtet man eine Kontraktion des Moleküls. Molvoluminamessungen der Äthylenisotope in flüssiger Phase ${ }^{6}$ haben gezeigt, daß eine Kontraktion von ca. $1,2 \%$ bzw. $0,5 \mathrm{~cm}^{3} / \mathrm{Mol}$ zwischen $\mathrm{C}_{2} \mathrm{H}_{4}$ und $\mathrm{C}_{2} \mathrm{D}_{4}$ auftritt. Nach BARTELL ${ }^{7}$ ist dies hauptsächlich ein intramolekularer Effekt und spiegelt die Volumenkontraktion der einzelnen Moleküle wieder. Bei der intermolekularen Wechselwirkung wird sich dieser Effekt als eine Verkleinerung von $\sigma$ bemerkbar machen.

Eine weitere Folge der Erniedrigung der Schwingungsniveaus ist eine Verringerung der Polarisierbarkeit, die sich in einer Verringerung von $\varepsilon$ äußert. Messungen von Polarisierbarkeiten haben Effekte in dieser Richtung bestätigt, jedoch liegen keine Daten für die Isotope $\mathrm{C}_{2} \mathrm{H}_{4}-\mathrm{C}_{2} \mathrm{D}_{4}$ vor.

Zur Bestimmung der Isotopie-Effekte in $\varepsilon$ und $\sigma$,

$$
\delta=\frac{\varepsilon^{\prime}-\varepsilon}{\varepsilon^{\prime}}, \quad \varrho=\frac{\sigma^{\prime}-\sigma}{\sigma^{\prime}}
$$

reichen die Daten aus Isotopie-Effekten der zweiten Virialkoeffizienten nicht aus. Diese liefern lediglich eine Beziehung zwischen $\delta$, $\varrho$ und $\left(B^{\prime}-B\right) / B^{\prime}$. Zur Ermittlung von $\delta$ und $\varrho$ sind zusätzliche Daten über $\varrho$ oder $\delta$ erforderlich.

\footnotetext{
${ }^{6}$ J. Bigeleisen, Th. Dorfmüller u. F. Menes, noch nicht veröffentlicht.
}

7 S. Bartell u. R. Roskos, J. Chem. Phys. 44, 457 [1966]. 
Die Beziehung (2) wurde unter Verwendung des Lennard-Jones-Potentials (3) integriert. Geht man auf die reduzierten Größen

$$
T^{*}=\frac{T}{\varepsilon / k} \quad \text { und } \quad B^{*}=\frac{B}{\frac{2}{3} \pi N \sigma^{3}}=\frac{B}{b_{0}}
$$

über, so erhält man :

$$
B^{*}\left(T^{*}\right)=\sum_{j=0}^{\infty} b^{(j)} T^{*-(2 j+1) / 4} .
$$

Die Faktoren $b^{(j)}$ werden durch die $\Gamma$-Funktionen gegeben :

$$
b^{(j)}=-\frac{2^{j+\frac{1}{2}}}{4 j !} \Gamma\left(\frac{2 j-1}{4}\right) .
$$

Unter den Voraussetzungen, daß :

a) Die Virialkoeffizienten der Isotope dem Theorem der Übereinstimmenden Zustände genügen,

b) $\varrho \ll 1, \delta \ll 1$ ist,

kann die Beziehung

$$
\frac{B^{\prime}-B}{B^{\prime}}=3 \varrho-\frac{-\delta S_{1}\left(T^{\prime *}\right)+\frac{1}{2} \delta^{2} S_{2}\left(T^{* *}\right)}{b_{0}^{\prime}}
$$

abgeleitet werden. Hierin ist:

$$
\begin{aligned}
S_{1}\left(T^{*}\right) & =\sum_{j=0}^{\infty} b^{(j)} \frac{2 j+1}{4} T^{*-(2 j+1) / 4}, \\
S_{2}\left(T^{* *}\right) & =\sum_{j=0}^{\infty} b^{(j)} \frac{2 j+1}{4} \frac{2 j+5}{4} T^{*-(2 j+1) / 4}, \\
b_{0}{ }^{\prime} & =\frac{2}{3} \pi N \sigma^{3} .
\end{aligned}
$$

Die Beziehung (4) liefert den gesuchten Zusammenhang zwischen $\delta, \varrho$ und $\left(B^{\prime}-B\right) / B^{\prime}$.

Eine Berechnung der Faktoren $S_{1}$ und $S_{2}$ für $T^{*}=1,185$ entsprechend der Meßtemperatur von $236,04{ }^{\circ} \mathrm{K}$ liefert

$$
\left(B^{\prime}-B\right) / B^{\prime}=3 \varrho-0,025 \delta+0,031 \delta^{2} .
$$

In diesem Temperaturbereich ist $\left(B^{\prime}-B\right) / B^{\prime}$ im wesentlichen von dem Isotopie-Effekt in $\varrho$ abhängig. Berücksichtigt man den oben geschätzten TranslationsQuanteneffekt von $0,12 \mathrm{~cm}^{3} / \mathrm{Mol}$, so ist der auf die Änderung des Paarpotentials zurückzuführende Effekt $\left(\left(B^{\prime}-B\right) / B\right)_{\text {pot }}=1,12 \cdot 10^{-2}$, und somit ergibt sich $Q \cong 0,37 \cdot 10^{-2}$.

Dieses Ergebnis läßt sich mit $\varrho$-Werten aus Molvolumina-Isotopie-Effekten in flüssiger Phase vergleichen ${ }^{6}$. In dem Temperaturbereich zwischen 130 und $160^{\circ} \mathrm{K}$ ergibt sich für den Isotopie-Effekt zwischen $\mathrm{C}_{2} \mathrm{H}_{4}$ und $\mathrm{C}_{2} \mathrm{D}_{4}: \quad \varrho=0,4 \cdot 10^{-2}$. Die Übereinstimmung ist recht gut und bestätigt das Ergebnis, daß der Effekt bei $T^{*} \geqq 1$ hauptsächlich ein Volumeneffekt ist. Weitere Messungen sind im Gange zur Untersuchung der Temperaturabhängigkeit des Effekts, insbesondere bei tiefen Temperaturen, bei denen Quanteneffekte wirksam werden und die Terme $\delta S_{1}$ und $\frac{1}{2} \delta^{2} S_{2}$ ins Gewicht fallen.

Der Deutschen Forschungsgemeinschaft danken wir für die finanzielle Unterstützung (W. G.) und die großzügige Bereitstellung von Sachmitteln. Herrn Prof. R. Haul sind wir für die Förderung dieser Arbeit sehr zu Dank verpflichtet.

\section{Umladungs- und Ionisationswirkungsquerschnitte beim Stoß von Protonen auf $\mathrm{He}-3$ und $\mathrm{He}-4$}

\section{Mark Becker und Arthur Scharmann}

I. Physikalisches Institut der Universität Gießen

(Z. Naturforsch. 24 a, 854-855 [1969] ; eingegangen am 31. März 1969)

Die Umladungs- und Ionisationswirkungsquerschnitte beim $\mathrm{Sto} ß$ von $\mathrm{H}^{+}$-, $\mathrm{D}^{+}$-, $\mathrm{H}_{2}{ }^{+}$- und $\mathrm{D}_{2}{ }^{+}$-Ionen auf leichten und schweren Wasserstoff $\left(\mathrm{H}_{2}\right.$ und $\left.\mathrm{D}_{2}\right)$ wurden von HOLLRICHeR ${ }^{1,2}$ mit der Kondensatormethode gemessen. Er fand im Energiebereich von 1,5 bis $30 \mathrm{keV}$ für die Umladungsquerschnitte beim Beschuß mit Atomionen den größten Isotopieeffekt, der bei gleicher Ionengeschwindigkeit folgende Abhängigkeit zeigte:

$$
\sigma\left(\mathrm{H}^{+}, \mathrm{D}_{2}\right)>\sigma\left(\mathrm{H}^{+}, \mathrm{H}_{2}\right) \approx \sigma\left(\mathrm{D}^{+}, \mathrm{D}_{2}\right)>\sigma\left(\mathrm{D}^{+}, \mathrm{H}_{2}\right) .
$$

Die maximalen Unterschiede betrugen bis zu knapp 10\%. Neuere Untersuchungen wurden von МЕсквасH et al. ${ }^{3}$ beim Stoß von Protonen und Deuteronen auf leichten und schweren Wasserstoff $\left(\mathrm{H}_{2}\right.$ und $\left.\mathrm{D}_{2}\right)$ mit

1 O. Hollricher, Dissertation, Gießen 1964.

2 O. Hollricher, Z. Physik 187, 41 [1965]. der Ladungsausgleichsmethode ausgeführt. Aus den einzelnen Ladungsgleichgewichtsanteilen wurden nach Allison ${ }^{4}$ die Wirkungsquerschnitte berechnet. Es wurde kein Unterschied bei den Wirkungsquerschnitten verschiedener Isotopenkombinationen bei gleicher Ionengeschwindigkeit gefunden, obwohl die Meßgenauigkeit von $1-2 \%$ größer als bei HolLRICHER war. Eine theoretische Abschätzung des Problems $\mathrm{H}^{+}+\mathrm{H}$ $\rightarrow \mathrm{H}+\mathrm{H}^{+}$von $\mathrm{PONCE}^{3}$ bestätigt die experimentellen Ergebnisse von МесквACH et al.

Der Widerspruch zwischen den Messungen von HollRICHER und MeсквACH et al. bei den Wirkungsquerschnitten sollte nicht von der Verwendung verschiedener Meßverfahren abhängen. Deshalb wurde der Isotopieeffekt bei den hier besprochenen Messungen an einem anderen Atomsystem unter Benutzung der gleichen, nur wenig veränderten Apparatur und des gleichen Meßverfahrens wie bei HolLRICHER überprüft. Es wurde mit Protonen einer Energie von 3 bis $30 \mathrm{keV}$ auf He-3 und He-4 geschossen. Nach den Ergebnissen von Hollricher müßte man hier zumindest bei den Umladungswirkungsquerschnitten einen meßbaren Isotopieeffekt erwarten.

3 W. Meckbach, G. Lantscher, V. H. Ponce u. E. SalvaTELLI, persönliche Mitteilung 1968.

4 S. K. Allison, Rev. Mod. Phys. 30, 4 [1958]. 